\title{
Valorización energética de residuos orgánicos mediante pirolisis
}

\section{Energy valorization of organic waste through pyrolysis}

\author{
Wildor Gosgot Angeles ${ }^{1 *}(\mathbb{D})$, Rosalynn Yohanna Rivera López ${ }^{1}$ (D), Jesús Rascón ${ }^{1}$ (D), Miguel Barrena Gurbillón ${ }^{1}$ (D), Carla
} María Ordinola Ramirez ${ }^{1}$ (D), Manuel Oliva ${ }^{1}$ (D), Yesica Montenegro Santillan ${ }^{1}$ (iD

\section{RESUMEN}

En la actualidad el aprovechamiento de energía de la biomasa es una alternativa para el impulso de matriz energética renovable. Una de las principales fuentes de biomasa son los residuos orgánicos generados por las actividades productivas y cotidianas. Por lo que existen diferentes mecanismos para valorización energéticas como tratamientos biológicos y termoquímicos. Debido a ello, la presente revisión se centra en las tecnologías termoquímicas, en especial en la pirólisis, ya que con este proceso se puede transformar todo tipo de residuos orgánicos, obteniendo productos principales como los bio-aceites, biochares y gases condensables que tienen un alto valor energético, que pueden ser aprovechados en los sistemas de calefacción y generación de electricidad, entre otras aplicaciones. Para obtener cada uno de los productos y aplicaciones dependerá del tipo de pirólisis y el reactor pirolítico. Estos son seleccionados de acuerdo a la composición de la biomasa a procesar. En definitiva, la pirólisis es una tecnología amigable con el medio ambiente debido a que se desarrolla en ausencia de oxígeno y no genera la emisión de gases de efecto invernadero causantes del cambio climático, además que, el biochar, producto de la pirólisis, ha despertado gran interés desde el punto de vista energético y remediación ambiental por la comunidad científica.

Palabras clave: biomasa, energía, biochar, ambiente.

\begin{abstract}
At present, the use of biomass energy is an alternative for the promotion of renewable energy matrix. One of the main sources of biomass are organic waste generated by productive and daily activities. So there are different mechanisms for energy recovery such as biological and thermochemical treatments. Due to this, this review focuses on thermochemical technologies, especially pyrolysis, since with this process all types of organic waste can be transformed, obtaining main products such as bio-oils, char and condensable gases that have a high energy value, which can be used in heating systems, electricity generation, among other applications. To obtain each of the products and applications depends on the type of pyrolysis and the pyrolytic reactor, these are selected according to the composition of the biomass to be processed. Therefore, pyrolysis is an environmentally friendly technology because it develops in the absence of oxygen and does not generate the emission of greenhouse gases that cause climate change, in addition to char, the product of pyrolysis, it has aroused great interest from an energy standpoint and environmental remedy for the scientific community.
\end{abstract}

Keywords: biomass, energy, biochar, environment.

\footnotetext{
${ }^{1}$ Universidad Nacional Toribio Rodríguez de Mendoza de Amazonas, Instituto de Investigación para el Desarrollo Sustentable de Ceja de Selva, Chachapoyas, Perú

"Autor de correspondencia. E-mail: wildor.gosgot@untrm.edu.pe
} 


\section{INTRODUCCIÓN}

Actualmente se generan grandes volúmenes de residuos sólidos provenientes de múltiples sectores como el doméstico, comercial, industrial y de construcción, todos ellos producto de las actividades antropogénicas (Elkhalifa et al., 2019). El mayor porcentaje de la composición de los residuos generados es de residuos orgánicos, ya que un tercio de los alimentos producidos a nivel mundial son desechados, y estos no reciben tratamiento alguno. En consecuencia, se genera la emisión de gases de efecto invernadero (metano, vapor de agua y dióxido de carbono) durante su descomposición en el aire libre, representando una carga negativa para el ambiente (Corrado et al., 2019).

Para suplir esta deficiencia en la gestión de residuos orgánicos han surgido diferentes tecnologías de valorización. Las dos tecnologías más utilizadas son el compostaje y la digestión anaeróbica. Sin embargo, ambos procesos, especialmente el compostaje toma una cantidad considerable de tiempo incluso con el uso y el costo de los aceleradores enzimáticos, en este sentido los residuos municipales contienen cantidades significativas de biomasa lignocelulosa, que tienen un potencial considerable para ser aplicados como fuente de energía (Li et al., 2017).

Hoy en día la producción de biocombustibles se presenta como una alternativa para lograr una mejor seguridad energética a partir de desechos orgánicos, controlando la contaminación, a través de una producción sostenible y mejoras sociales (Stephen y Periyasamy, 2018). En este enfoque la pirólisis es una tecnología que ha sido ampliamente estudiada y desarrollada en todo el mundo como un proceso práctico en la valorización de residuos agrícolas, forestales e industriales (Mattos et al., 2019), para convertirlas de manera eficiente en biocombustibles (Elkhalifa et al., 2019). Por ello, este trabajo está enfocado en revisar el desarrollo actual y evaluar las oportunidades potenciales para la pirólisis de residuos orgánicos desde el punto de vista energético.
I I. VALORIZACIÓN DE RESIDUOS ORGÁNICOS

Los residuos sólidos generados en las actividades productivas y de consumo constituyen un potencial recurso económico, por lo tanto, la valorización es una alternativa que considera su utilidad en actividades de reciclaje de sustancias inorgánicas y metales, generación de energía, producción de compost, fertilizantes u otras transformaciones biológicas, recuperación de componentes, tratamiento o recuperación de suelos, entre otras opciones que eviten su disposición final. Todasestas consideraciones están establecidas en el DS 1278-2017- MINAM, Ley de Gestión Integral de residuos sólidos.

\section{TECNOLOGÍAS DE VALORACIÓN ENERGÉTICA DE LOS RESIDUOS}

Los objetivos de cualquier sistema de gestión de residuos son la valorización de materiales, energía y la correcta eliminación de los residuos con la finalidad de reducir las emisiones de gases de efecto invernadero e impactos negativos al ambiente (Cerdá, 2012). La tecnología de valoración se elige no solo en función a los costos que esta demande, a la recuperación de energía, o a la capacidad de degradación del residuo, sino también orientada a la búsqueda de la sostenibilidad ambiental durante el proceso de tratamiento $(\mathrm{Ku}-$ mar y Samadder, 2017).

Dentro de las tecnologías que se utilizan para la valorización energética encontramos el tratamiento biológico y/o térmico de los residuos. Los tratamientos biológicos incluyen el compostaje y la digestión anaerobia, mientras que en las tecnologías térmicas tenemos la incineración, gasificación y pirólisis. Los residuos que no pueden reciclarse ni tratarse se eliminan en rellenos sanitarios, en los cuales la descomposición de los residuos genera un gas que puede ser aprovechado energéticamente (Sipra et al., 2018; Sindhu et al., 2019).

Las propiedades fisicoquímicas y el mecanismo de transformación de los residuos de biomasa pueden producir diferentes productos de valor agregado. Por ejemplo, la biomasa oleaginosa se trata por esterifica- 
ción (Ácido carboxílico + Alcohol $\leftrightarrow$ Éster + Agua) y sacarificación (proceso de rotura de un carbohidrato complejo [almidón, celulosa] en sus componentes monosacáridos). Por otra parte, la biomasa lignocelulósica se puede procesar por combustión, pirólisis, hidrolisis, digestión anaeróbica, licuefacción y gasificación. (Dhyani y Bhaskar, 2018).

\section{TECNOLOGÍAS DE TRATAMIENTO TÉRMICO DE RESIDUOS SÓLIDOS}

Al aplicar las tecnologías de conversión térmica, los residuos pasan por procesos termoquímicos, endotérmicos y exotérmicos como son el secado, la desvolatilización, la reducción y la oxidación de volátiles (Pérez et al., 2010). Por ello, el modelado, diseño y la operación de los sistemas de conversión térmica es un desafío debido a la gran variedad de la composición de los residuos sólidos municipales y las diferencias en su comportamiento de degradación térmica (Sorum et al., 2001).

Las técnicas termoquímicas para la conversión de residuos sólidos se pueden clasificar en tres categorías generales: pirólisis, combustión y gasificación (Sindhu et al., 2019). La selección del método de conversión apropiado depende de los productos deseados, que en la mayoría de los casos deben maximizarse mientras se minimizan los gastos en el proceso e impactos negativos al ambiente (Elkhalifa et al., 2019).

\section{Combustión}

La combustión de residuos, conocida como quema masiva, es un proceso en los componentes dentro de los residuos que tienen una oxidación completa (Lombardi et al., 2015). Inicialmente, se consideraba la incineración como un proceso solo para la reducción de residuos, sin embargo, hoy en día esto ha dado un giro convirtiéndolo en una técnica para la recuperación de energía (Kumar and Samadder 2017). Un problema que surge de esta técnica es la corrosión por las elevadas temperaturas, por lo que las temperaturas de operación deben estar por debajo de $\operatorname{los} 1150^{\circ} \mathrm{C}$ (Mayer et al., 2019).

\section{Gasificación}

La gasificación es un proceso destinado a convertir un sólido a un combustible gaseoso, llamado "gas productor" o "syngas" (Lombardi et al., 2015). La gasificación es aplicable para todo tipo de residuos, porque no produce emisiones y reduce el volumen de los residuos entre un 50 y 90\% (Sipra et al., 2018).

Esta tecnología de conversión térmica que transforma residuos secos en combustibles de bajo poder calorífico, que puede ser utilizado en turbinas o equipos de generación de calor, debido a su bajo poder calorífico neutro en $\mathrm{CO}_{2}$, rico en nitrógeno, alquitrán y el coque (Mayer et al., 2019). Por otro lado, la gasificación también permite el aprovechamiento energético de los residuos verdes obteniendo productos energéticamente aprovechables.

\section{Pirólisis}

La pirólisis es la descomposición térmica de residuos en ausencia de oxígeno que produce productos gaseosos, líquidos y sólidos (Chhabra et al., 2016; Bridgwater, 2018). Esta tecnología permite la recuperación, facilita el transporte, almacenamiento y aprovechamiento de los residuos y, además, es de fácil y bajo costo de operación y mantenimiento (Czajczyńska et al., 2017; Zaman et al., 2017). Por ello, la pirólisis es considerada como una de las soluciones más sostenibles en la gestión y valorización de los residuos (Grycová et al., 2016).

\section{PIRÓLISIS DE RESIDUOS ORGÁNICOS}

La pirólisis es una de las tecnologías de conversión térmica más prometedoras debido a que es un método utilizado para la producción de energía y productos químicos a partir de la composición carbonosa de la biomasa residual (Kwon et al., 2019).

La pirólisis convierte la biomasa en tres subproductos: líquidos (bio-aceite), sólido (bio-carbón) productos gaseosos que comprenden $\mathrm{CO}_{2}, \mathrm{CO}, \mathrm{H}_{2}$, $\mathrm{CH}_{4}$ (Chen et al., 2018; Hu y Gholizadeh, 2019).

Según Chen et al. (2015), las reacciones que se llevan a cabo dentro de la pirólisis para la formación de los productos (biochar, bioaceite y gas), se establecen según la ecuación: 
$\mathrm{C}_{\mathrm{x}} \mathrm{H}_{\mathrm{y}} \mathrm{O}_{\mathrm{z}}+\mathrm{Q} \rightarrow$ biochar+bioaceite + gas $+\mathrm{H}_{2} \mathrm{O}$

Donde, $Q$ es el calor que necesita ser ingresado al reactor para que tengan lugar las reacciones.

\section{Tipos de pirólisis de residuos orgánicos}

Torrefacción

La torrefacción es un pre-tratamiento termoquímico utilizado en la descomposición de materia orgánica, por lo general de origen lignocelulosa (Arteaga Pérez et al., 2016). Las velocidades de calentamiento que se emplean en la torrefacción son lentas y prolongadas. Su función es lograr la pérdida de agua de la biomasa tratada (Daful \& Chandraratne, 2018), por lo tanto, este proceso térmico de conversión de biomasa modifica la composición fisicoquímica como peso, contenido de carbono, y cenizas entre otros (Kim et al., 2012). El contenido de humedad y de oxígeno disminuyen, mientras que el contenido de carbono aumenta mejorando la capacidad de combustible (Chen et al., 2018).

\section{Pirólisis lenta}

La pirólisis lenta es la exposición de la biomasa residual a temperaturas moderadas por un periodo de tiempo prolongado en ausencia de oxígeno (Daful y Chandraratne, 2018). La pirólisis lenta se aplica en un intervalo de contenido de humedad entre $15 \%$ y $20 \%$ para obtener el carbón vegetal conocido como biochar como principal producto del proceso térmico (Dhyani y Bhaskar, 2018).

\section{Pirólisis rápida}

La pirólisis rápida está caracterizada por una temperatura de operación alta junto con una velocidad de calentamiento rápida, la cual aumenta el rendimiento y calidad del bio-aceite como su principal producto (Chhabra et al., 2016).

\section{Pirólisis ultrarrápida}

Se la conoce también como pirólisis "flash" por la velocidad de calentamiento, obteniendo como producto bio-aceites en un $75 \%$ (Klug, 2012). Este procedimiento se lleva a cabo mediante una rápida desvolatilización en atmósfera inerte utilizando una velocidad de calentamiento más alta con temperaturas alrededor de 450 y $1000^{\circ} \mathrm{C}$ (Zaman et al., 2017).

La condición de operación y los productos obtenidos de acuerdo a los tipos de pirólisis se describen en el Tabla 1.

Tabla 1. Condiciones de operación de diferentes tipos de pirólisis

\begin{tabular}{|c|c|c|c|c|c|}
\hline Tipo & Condición & $\begin{array}{c}\text { Líquidos (bio- } \\
\text { aceites) }\end{array}$ & $\begin{array}{c}\text { Solidos } \\
\text { (biochar) }\end{array}$ & $\begin{array}{c}\text { Gas } \\
\text { (syngas) } \\
\end{array}$ & $\begin{array}{l}\text { Velocidad de } \\
\text { calentamiento }\end{array}$ \\
\hline Torrefacción & $\begin{array}{c}\left(<300{ }^{\circ} \mathrm{C}\right) \\
\text { tiempo de } \\
\text { exposición } \\
>2 \mathrm{~h})\end{array}$ & $20 \%$ & $75 \%$ & $5 \%$ & $<1^{\circ} \mathrm{C} / \mathrm{s}$ \\
\hline Pirólisis lenta & $\begin{array}{l}\left(300-550^{\circ} \mathrm{C}\right) \\
\text { tiempo de } \\
\text { resistencia } \\
\text { horas o días) } \\
\left(425-600^{\circ} \mathrm{C}\right)\end{array}$ & $\begin{array}{c}30 \% \quad(70 \% \\
\text { agua })\end{array}$ & $35 \%$ & $35 \%$ & $1-1.8^{\circ} \mathrm{C} / \mathrm{s}$ \\
\hline Pirólisis rápida & $\begin{array}{l}\text { Tiempo de } \\
\text { resistencia del } \\
\text { vapor }(<2 \mathrm{~s})\end{array}$ & $\begin{array}{c}75 \%(25 \% \\
\text { agua })\end{array}$ & $12 \%$ & $13 \%$ & $10-1000^{\circ} \mathrm{C} / \mathrm{s}$ \\
\hline Pirólisis flash & $\begin{array}{c}\left(750-1000^{\circ} \mathrm{C}\right) \\
(0.5 \mathrm{~s})\end{array}$ & - & & & $>1{ }^{\circ} \mathrm{C}$ \\
\hline
\end{tabular}

Fuete: Daful y Chandraratne (2018)

\section{Tipos de reactores de pirólisis de residuos orgánicos}

Los reactores han sido diseñados de tal manera que satisfacen condiciones específicas teniendo en cuenta parámetros como la temperatura de calentamiento, el tiempo de residencia del producto de vapor y la pre- sión requerida, para un alto rendimiento de bio-aceite (Zaman et al., 2017). En consideración de lo anterior, los investigadores han desarrollado diferentes tipos de reactores que se muestran en la Tabla 2. 
Tabla 2. Tipos de reactores de pirólisis

\begin{tabular}{|c|c|c|c|}
\hline $\begin{array}{l}\text { Tipo de } \\
\text { reactor }\end{array}$ & Descripción & Ventajas & Desventajas \\
\hline Lecho fijo & $\begin{array}{l}\text { Está compuesto por un } \\
\text { compartimiento de enfriamiento } \\
\text { de gases y un sistema de } \\
\text { limpieza mediante filtración a } \\
\text { través de ciclones, depuradores } \\
\text { húmedos y filtros secos }\end{array}$ & Simplicidad en el diseño & $\begin{array}{l}\text { Alta conservación de } \\
\text { carbono. Tiempo de } \\
\text { residencia sólido } \\
\text { prolongado } \\
\text { Baja carga de cenizas } \\
\text { Difícil de eliminar. }\end{array}$ \\
\hline $\begin{array}{l}\text { Lecho } \\
\text { fluidizado } \\
\text { burbujeante }\end{array}$ & $\begin{array}{l}\text { La velocidad del agente } \\
\text { fluidizante-gasificante es } \\
\text { suficientemente baja para que } \\
\text { no haya una circulación } \\
\text { significativa de sólido. }\end{array}$ & $\begin{array}{l}\text { Diseño simple } \\
\text { Procedimientos } \\
\text { operativos sencillos }\end{array}$ & $\begin{array}{l}\text { Se necesitan pequeños } \\
\text { tamaños de partículas }\end{array}$ \\
\hline $\begin{array}{l}\text { Lecho } \\
\text { fluidizado } \\
\text { circulante }\end{array}$ & $\begin{array}{l}\text { La velocidad del agente es } \\
\text { mucho más elevada, dando } \\
\text { como resultado una circulación } \\
\text { de sólidos. Este sólido es } \\
\text { separado de la corriente gaseosa } \\
\text { y recirculado al reactor por } \\
\text { medio del uso de un ciclón y un } \\
\text { sistema de retorno al } \\
\text { gasificador }\end{array}$ & Mejor control térmico & $\begin{array}{l}\text { Producción a gran escala } \\
\text { difícil }\end{array}$ \\
\hline Cono giratorio & $\begin{array}{l}\text { El reactor de cono giratorio } \\
\text { requiere que la mezcla de } \\
\text { biomasa y arena caliente se } \\
\text { realice mecánicamente y no } \\
\text { requiere el uso de gas inerte. }\end{array}$ & $\begin{array}{l}\text { La fuerza centrífuga } \\
\text { circula arena caliente y } \\
\text { sustrato de biomasa. } \\
\text { No se requiere gas } \\
\text { portador }\end{array}$ & $\begin{array}{l}\text { Proceso operativo difícil } \\
\text { La aplicación a gran escala } \\
\text { es difícil }\end{array}$ \\
\hline Vacío & $\begin{array}{l}\text { El diseño es muy complicado y } \\
\text { los requisitos de inversión y } \\
\text { mantenimiento siempre son } \\
\text { altos, lo que hace que la } \\
\text { tecnología sea antieconómica. } \\
\text { La biomasa se transporta a la } \\
\text { cámara de vacío a alta } \\
\text { temperatura con la ayuda de } \\
\text { una cinta transportadora de } \\
\text { metal con agitación periódica } \\
\text { de la biomasa mediante } \\
\text { agitación mecánica }\end{array}$ & $\begin{array}{l}\text { El aceite está limpio. } \\
\text { Puede procesar partículas } \\
\text { más grandes de } 3-5 \mathrm{~cm} \text {. } \\
\text { No se requiere gas } \\
\text { portador }\end{array}$ & $\begin{array}{l}\text { Proceso lento } \\
\text { El tiempo de residencia } \\
\text { sólido es demasiado alto } \\
\text { Requiere equipo a gran } \\
\text { escala } \\
\text { Pobre tasa de transferencia } \\
\text { de calor y masa Genera más } \\
\text { agua }\end{array}$ \\
\hline Ablativo & $\begin{array}{l}\text { En este reactor, el } \\
\text { calentamiento se realiza a } \\
\text { través de una capa fundida en la } \\
\text { superficie del reactor caliente y } \\
\text { en ausencia de gas fluidizante }\end{array}$ & $\begin{array}{l}\text { No se requiere gas inerte } \\
\text { Se pueden procesar } \\
\text { partículas más grandes }\end{array}$ & $\begin{array}{l}\text { El sistema es más intensivo } \\
\text { El reactor es costoso }\end{array}$ \\
\hline Microonda & $\begin{array}{l}\text { La transferencia de energía } \\
\text { ocurre como resultado de la } \\
\text { interacción entre las moléculas y } \\
\text { los átomos usando microondas. } \\
\text { Todo el proceso de secado y } \\
\text { pirólisis se lleva a cabo en una } \\
\text { cámara de horno de microondas } \\
\text { conectada a una fuente de } \\
\text { electricidad. El gas portador es } \\
\text { inerte y también se usa para } \\
\text { crear una cámara libre de } \\
\text { oxígeno. }\end{array}$ & $\begin{array}{l}\text { Transferencia de calor } \\
\text { eficiente } \\
\text { Estructura compacta } \\
\text { Mayor velocidad de } \\
\text { calentamiento } \\
\text { Se puede procesar } \\
\text { biomasa de gran } \\
\text { tamaño } \\
\text { Distribución de } \\
\text { temperatura uniforme }\end{array}$ & $\begin{array}{l}\text { Alta temperatura } \\
\text { Alto consumo de energía } \\
\text { eléctrica } \\
\text { Altos costos de operación }\end{array}$ \\
\hline
\end{tabular}


Tabla 2. Continuación

\begin{tabular}{|c|c|c|c|}
\hline $\begin{array}{l}\text { Tipo de } \\
\text { reactor }\end{array}$ & Descripción & Ventajas & Desventajas \\
\hline Barrena & $\begin{array}{l}\text { Este reactor utiliza una barrena } \\
\text { para mover la muestra de } \\
\text { alimentación a través de un tubo } \\
\text { cilíndrico que se calienta y no } \\
\text { contiene oxígeno. }\end{array}$ & $\begin{array}{l}\text { No se requiere gas } \\
\text { portador }\end{array}$ & $\begin{array}{l}\text { Baja temperatura del proceso } \\
\text { Piezas móviles en zona } \\
\text { caliente }\end{array}$ \\
\hline Plasma & $\begin{array}{l}\text { Este reactor está compuesto por } \\
\text { un tubo de cuarzo que es } \\
\text { cilíndrico y está equipado con } \\
\text { dos electrodos de cobre. La } \\
\text { alimentación de la materia prima } \\
\text { se realiza en el medio utilizando } \\
\text { un tornillo con tornillo de } \\
\text { velocidad variable en la parte } \\
\text { superior del tubo }\end{array}$ & $\begin{array}{l}\text { Alta densidad de } \\
\text { energía } \\
\text { Alta transferencia de } \\
\text { calor } \\
\text { Control efectivo del } \\
\text { proceso } \\
\text { Alto consumo de } \\
\text { energía eléctrica }\end{array}$ & $\begin{array}{l}\text { Altos costos de operación } \\
\text { Se requieren pequeños } \\
\text { tamaños de partícula }\end{array}$ \\
\hline Solar & $\begin{array}{l}\text { Está formado por un tubo de } \\
\text { cuarzo con pared externa que es } \\
\text { opaca, generalmente expuesta a } \\
\text { una alta concentración de } \\
\text { radiación solar, capaz de generar } \\
\text { altas temperaturas }\left(>700^{\circ} \mathrm{C}\right) \text { en } \\
\text { el reactor }\end{array}$ & $\begin{array}{l}\text { Utilizar energía } \\
\text { renovable } \\
\text { Mayor tasa de } \\
\text { calentamiento }\end{array}$ & $\begin{array}{l}\text { Alta temperatura } \\
\text { Depende de las condiciones } \\
\text { del clima }\end{array}$ \\
\hline
\end{tabular}

Fuente: Zaman et al. (2017)

\section{Productos de pirólisis de residuos orgánicos \\ Gases}

El gas resultante de la pirólisis está compuesto por un $82,51 \%$ de gases combustibles y solo un $17,49 \%$ de dióxido de carbono (Cheng et al., 2019). Los gases resultantes como el óxido de carbono, monóxido de carbono y metano, dependen de la composición de la biomasa utilizada (Dhyani y Bhaskar, 2018).

\section{Bio-aceite}

El bio-aceite proveniente del proceso de pirólisis es un líquido marrón oscuro (Dhyani y Bhaskar, 2018). Este contiene cuatro veces más de potencial energético que la biomasa cruda, pudiendo ser utilizado en calefacción, generación de electricidad o extracción de sustancias químicas. Para lograrlo es necesario que la biomasa atraviese pre-tratamientos que garanticen un bio-aceite con alto valor de calentamiento, con bajo contenido en agua, en cenizas y acidez (AlvarezChavez et al., 2019).

La alta temperatura de operación disminuye el contenido carboxílico en el bio-aceite, es así que a mayor velocidad de calentamiento se obtiene mayor contenido de aromáticos y mayores compuestos nitrogenados (Tira- panampai et al., 2019). En este sentido la temperatura ideal en la producción de bio-aceite debe ser de $550^{\circ} \mathrm{C}$. Este producto es un combustible limpio y sostenible con uso significativo en la industria química en la elaboración de saborizantes para alimentos, fertilizantes y resina (Cai et al., 2019), Además no tiene contenido de azufre por lo tanto su uso no genera emisiones de óxido de azufre (Sukumar et al., 2020).

\section{Biochar}

El biochar o carbón vegetal es el residuo sólido rico en carbono, resultado de la pirolización de cualquier tipo de residuos sólidos orgánicos. Las características del biochar generado por pirólisis dependen del grado de temperatura y tiempo al que son expuestos (Rehrah et al., 2016).

En los últimos años el biochar viene teniendo intereses por las diversas aplicaciones ambientales (Oliveira et al., 2017), debido a que tiene la capacidad de: remediar suelos (mejorando su conductividad eléctrica y sus parámetros de fertilidad, reduciedo perdidas de nutrientes, aumentando la nitrificación), descontaminar cuerpos de agua (absorbiendo contaminantes inorgánicos), estabilizar $\mathrm{pH}$, descontaminar el aire, modi- 
ficar nutrientes, etc. (Rehrah et al., 2016); Sizmur et al., 2017). Los biochares también se utilizan en la mitigación del efecto invernadero (Watts et al., 2017).

En la actualidad, la aplicación innovadora del biochar se da en la absorción e inmovilización de metales pesa- dos como el cobre, plomo, cadmio y zinc (Wang y Liu, 2017; Godlewska et al., 2017)

\section{Aplicaciones de la pirólisis}

A continuación, en la Tabla 3 se describen las principales aplicaciones de la pirólisis:

Tabla 3. Aplicaciones de la pirólisis

\begin{tabular}{|c|c|c|c|}
\hline Biomasa & $\begin{array}{c}\text { Temperatura de } \\
\text { operación }\end{array}$ & $\begin{array}{c}\text { Productos obtenidos / } \\
\text { Rendimiento } \\
\end{array}$ & Referencias \\
\hline Tuza de maíz & $300{ }^{\circ} \mathrm{C}$ & Briquetas de biocarbón & Berastegui et al. ( 2017) \\
\hline & & Carbón vegetal $(27,5 \%)$ & \\
\hline Bagazo de caña & $440,81^{\circ} \mathrm{C}$ & Fracción líquida $(61,27 \%)$ & Penedo et al. (2008) \\
\hline & & Fracción de gas $(25,4 \%)$ & \\
\hline Esquilmos de palta & 360 a $700{ }^{\circ} \mathrm{C}$ & Biocarbón & Medina \& Medina (2018) \\
\hline \multirow{2}{*}{ Palma de aceite } & $600^{\circ} \mathrm{C}$ & Bio-aceite $(23.3 \%)$ & \multirow[b]{2}{*}{ Arteaga Pérez et al. (2016) } \\
\hline & $700^{\circ} \mathrm{C}$ & $\begin{array}{c}\text { Gases no codensablos de mayor } \\
\text { valor calorífico }\end{array}$ & \\
\hline Cáscara de la & \multirow{3}{*}{$550^{\circ} \mathrm{C}$} & Biocarbón & \multirow{3}{*}{$\begin{array}{l}\text { Martínez-Ángel et al. } \\
\text { (2015) }\end{array}$} \\
\hline mazorca de cacao & & Bio-aceites & \\
\hline \multirow{3}{*}{ Eucalipto } & & Bio-aceite (45\%) & \\
\hline & \multirow[t]{2}{*}{$500{ }^{\circ} \mathrm{C}$} & Bio-carbón (38\%) & \multirow[t]{2}{*}{ Félix et al. (2017) } \\
\hline & & Bio-gas (17\%) & \\
\hline Cuesco de palma & $500^{\circ} \mathrm{C}$ & $\begin{array}{l}\text { Material carbonizado }(31,14 \\
\mathrm{MJ} / \mathrm{kg})\end{array}$ & Romero et al. (2016) \\
\hline $\begin{array}{l}\text { Eucalyptus grandis } \\
\text { Acacia magnium } \\
\text { Gmelina arbórea }\end{array}$ & $700^{\circ} \mathrm{C}$ & Biochar & $\begin{array}{l}\text { Suárez-Hernández et al. } \\
\text { (2017) }\end{array}$ \\
\hline
\end{tabular}

\section{Consideraciones ambientales de la pirólisis}

El tratamiento de los residuos sólidos orgánicos por pirólisis es una alternativa sostenible de gestionar los residuos. Al mismo tiempo genera un valor agregado con las diferentes aplicaciones que se le puede dar a sus productos (Rehrah et al., 2016).

Debido a que la composición de los residuos varía de una zona a otra, los pretratamientos como el triturado y secado son necesarios, teniendo en consideración que los residuos orgánicos sometidos a pirólisis no deben contener un grado de humedad elevado (Sipra et al., 2018).

El tiempo requerido para tratar los desechos térmicamente toma de minutos $\mathrm{u}$ horas, $\mathrm{y}$ transforma los residuos en productos sin olor, libres de patógenos. Sin embargo, una de las limitaciones que tiene la aplicación de la tecnología de pirólisis es la necesidad de homogeneidad en los residuos a procesar (Lombardi et al., 2015).

\section{Comparación de las tecnologías térmicas de resi- duos sólidos}

Las tecnologías de descomposición termoquímicas están diferenciadas por la temperatura de operación, productos que generan, condiciones atmosféricas en las que se llevan a cabo y de la composición de los residuos que se procesan (Kumar y Samadder, 2017).

La comparación de las tecnologías de conversión termoquímicas se realiza en función de sus ventajas y desventajas donde la pirólisis se concluye como el proceso más respetuoso con el medio ambiente, basado en sus emisiones producidas (Tabla 4) (Sipra et al., 2018). Sin embargo, la selección la tecnología de conversión para el caso de residuos orgánicos está sujeta también a la fuente de biomasa residual y los productos energéticos que se desee obtener (Tripathi et al., 2016). 
Tabla 4. Comparación de tecnologías termoquímicas de conversión de residuos

\begin{tabular}{|c|c|c|c|c|}
\hline \multicolumn{2}{|c|}{ Parámetros } & Incineración & Pirólisis & Gasificación \\
\hline \multicolumn{2}{|c|}{ Principio } & $\begin{array}{l}\text { Combustión oxidativa } \\
\text { completa }\end{array}$ & $\begin{array}{l}\text { Degradación térmica de } \\
\text { residuos en ausencia de } \\
\text { oxígeno }\end{array}$ & Oxidación parcial \\
\hline \multicolumn{2}{|c|}{$\begin{array}{l}\text { Temperatura de operación } \\
\left({ }^{\circ} \mathrm{C}\right)\end{array}$} & $850-1200$ & $300-800$ & $800-1600$ \\
\hline \multicolumn{2}{|c|}{ Atmósferas } & $\begin{array}{c}\text { Presencia de suficiente } \\
\text { oxigeno }\end{array}$ & Ausencia de oxígeno & $\begin{array}{l}\text { Suministro } \\
\text { controlado de } \\
\text { oxígeno }\end{array}$ \\
\hline \multirow{3}{*}{ Productos } & Sólido & $\begin{array}{l}\text { Cenizas, escorias y otros } \\
\text { residuos no combustibles }\end{array}$ & Biochar y cenizas & Cenizas y escorias \\
\hline & Líquido & & Bio-aceites & \\
\hline & Gas & $\mathrm{CO}_{2}, \mathrm{H}_{2} \mathrm{O}, \mathrm{O}_{2}, \mathrm{~N}_{2}$ & $\begin{array}{l}\text { Gas de pirólisis }\left(\mathrm{H}_{2}, \mathrm{CO},\right. \\
\left.\text { Hidrocarburos, } \mathrm{H}_{2} \mathrm{O} \text { y } \mathrm{N}_{2}\right)\end{array}$ & $\begin{array}{c}\text { Syngas }\left(\mathrm{H}_{2}, \mathrm{CO},\right. \\
\mathrm{CO}_{2}, \mathrm{CH}_{4}, \mathrm{H}_{2} \mathrm{O} \text { y } \\
\left.\mathrm{N}_{2}\right)\end{array}$ \\
\hline \multicolumn{2}{|c|}{ Pretratamientos } & No requerido & Requerido & Requerido \\
\hline
\end{tabular}

Fuente: Kumary Samadder(2017)

\section{CONCLUSIONES}

La pirólisis es un método de valorización de los desechos de biomasa amigable con el medio ambiente en comparación con las técnicas bioquímicas y termoquímicas, debido a que se desarrolla en ausencia de oxígeno y no genera la emisión de gases de efecto invernadero causantes del cambio climático. Dentro de la tecnología de pirólisis, existen tres tipos: torrefacción, pirólisis lenta y pirólisis rápida, las cuales se caracterizan por la temperatura del proceso y el tipo de producto obtenido. Con respecto a la torrefacción se realiza en temperaturas menores a $300{ }^{\circ} \mathrm{C}$, obteniendo como producto biomasa seca. La pirólisis lenta en intervalos de temperatura de $300-550{ }^{\circ} \mathrm{C}$, obteniendo biochar y bio-aceites. Finalmente, la pirólisis rápida se desarrolla en intervalos de temperatura de $425-600^{\circ} \mathrm{C}$, obteniendo principalmente bio-aceites.

Los productos obtenidos del proceso de pirólisis aprovechando todo tipo de residuos orgánicos son de alto valor energético, a los que se les atribuye múltiples funcionalidades, entre ellas el aprovechamiento en sistemas de calefacción y generación de electricidad, entre otros. El biochar, producto principal de la pirólisis lenta, genera interés en la comunidad científica debido a las múltiples aplicaciones ambientales que se le atribuyen como mejorador de las características fisicoquímicas del suelo, descontaminador de agua, absorbente e inmovilizador de metales pesados, de aire y estabilizador de $\mathrm{pH}$.

\section{CONTRIBUCIÓN DE LOS AUTORES}

Todos los autores participaron en la redacción del manuscrito inicial, revisión bibliográfica, y en la revisión y aprobación del manuscrito final.

\section{CONFLICTO DE INTERESES}

Los autores declaran no tener conflicto de intereses.

\section{REFERENCIAS BIBLIOGRÁFICAS}

Alvarez-Chavez, B. J., S. Godbout, J. H. PalaciosRios, É. Le Roux, y V. Raghavan. 2019. "Physical, Chemical, Thermal and Biological Pre-Treatment Technologies in Fast Pyrolysis to Maximize Bio-Oil Quality: A Critical Review." Biomass and Bioenergy 128 (8): $\begin{array}{llllll}1 & 0 & 5 & 3 & 3 & 3\end{array}$. DOI:10.1016/j.biombioe.2019.105333.

Arteaga Pérez, L., C. Segura, y K. Diéguez Santana. 2016. "Procesos de Torrefacción Para Valorización de Residuos Lignocelulósicos. Análisis de Posibles Tecnologías de Aplicación En Sudamérica." Afinidad: Revista de Química Teórica y Aplicada 73 (573): 60-68.

Berastegui, B. C., R. J. P. Ortega, F. J. M. Mendoza, D. Y. E. González, y V. R. D. Gómez. 2017. "Elaboración de Biocombustibles Sólidos Densificados a Partir de Tusa de Maíz, Bioaglomerante de Yuca y Carbón Mineral Del Departa- 
mento de Córdoba." Ingeniare 25 (4):

$643-53$. D O I : 10.4067 / S 0718 33052017000400643.

Bridgwater, T. 2018. “Challenges and Opportunities in Fast Pyrolysis of Biomass: Part I." Johnson Matthey Technology Review 62 (1): 118-30. DOI:10.1595/205651318X696693.

Cai, W., N. Kang, M. Ki Jang, C. Sun, R. Liu, y Z. Luo. 2019. "Long Term Storage Stability of BioOil from Rice Husk Fast Pyrolysis.” Energy 186 : $\begin{array}{llllll}1 & 1 & 5 & 8 & 8 & 2\end{array}$. DOI:10.1016/j.energy.2019.115882.

Cerdá, E. 2012. "Energía Obtenida a Partir de Biomasa." Cuadernos Económicos de ICE 1 (83): 117-140. DOI:10.32796/cice.2012.83.6036.

Chen, D., L. Yin, H. Wang, y P. He. 2015. "Reprint of: Pyrolysis Technologies for Municipal Solid Waste: A Review." Waste Management 37: $\begin{array}{llllll}1 & 1 & 6 & - & 3 & 6\end{array}$. DOI:10.1016/j.wasman.2015.01.022.

Chen, Z., M. Wang, E. Jiang, D. Wang, K. Zhang, Y. Ren, y Y. Jiang. 2018. "Pyrolysis of Torrefied Biomass." Trends in Biotechnology 36 (12): $\begin{array}{lllllll}1 & 2 & 8 & 7 & - & 9 & 8\end{array}$. DOI:10.1016/j.tibtech.2018.07.005.

Cheng, S., J. Shu, H. Xia, S. Wang, L. Zhang, J. Peng, C. Li, X. Jiang, y Q.Zhang. 2019. "Pyrolysis of Crofton Weed for the Production of Aldehyde Rich Bio-Oil and Combustible Matter Rich Bio-Gas." Applied Thermal Engineer$i n g$, $1164-70$. DOI:10.1016/j.applthermaleng.2018.12.009.

Chhabra, V., Y. Shastri, y S. Bhattacharya. 2016. "Kinetics of Pyrolysis of Mixed Municipal Solid Waste-A Review." Procedia Environmental Sciences 35 : $513-27$. DOI:10.1016/j.proenv.2016.07.036.

Corrado, S., C. Caldeira, M. Eriksson, O. Jørgen Hanssen, H. Eduard Hauser, F. van Holsteijn, G. Liu, et al. 2019. "Food Waste Accounting Methodologies: Challenges, Opportunities, y Further Advancements." Global Food Secu-
$r i t y$
20 :
$93-100$.

DOI:10.1016/j.gfs.2019.01.002.

Czajczyńska, D., T. Nannou, L. Anguilano, R. Krzyzyńska, H. Ghazal, N. Spencer, y H. Jouhara. 2017. "Potentials of Pyrolysis Processes in the Waste Management Sector." Energy Procedia 123: 387-94. DOI:10.1016/j.egypro.2017.07.275.

Daful, A. G, y M. R. Chandraratne. 2018. "Biochar Production From Biomass Waste-Derived Material. Reference Module in Materials Science and Materials Engineering". Encyclopedia of Renewable and Sustainable Materials 4: 370-378. DOI:10.1016/b978-0-12803581-8.11249-4.

Dhyani, V. y T. Bhaskar. 2018. “A Comprehensive Review on the Pyrolysis of Lignocellulosic Biomass." Renewable Energy 129: 695-716. DOI:10.1016/j.renene.2017.04.035.

Elkhalifa, S., T. Al-Ansari, H. R. Mackey, y G. McKay. 2019. "Food Waste to Biochars through Pyrolysis: A Review." Resources, Conservation and Recycling 144:310-20. DOI:10.1016/j.resconrec.2019.01.024.

Félix, C. R. de O., A. F. de Azevedo Júnior, C. Costa Freitas, C. Augusto de Moraes Pires, V. Teixeira, R. Frety, y S. Teixeira Brandão. 2017. "Pirólise Rápida de Biomassa de Eucalipto $\mathrm{Na}$ Presença de Catalisador Al-MCM-41.” Revista Materia 22. DOI:10.1590/s1517707620170005.0251 .

Godlewska, P., H. Peter, Y. Sik, y P. Oleszczuk. 2017. "Bioresource Technology Biochar for Composting Improvement and Contaminants Reduction . A Review." Bioresource Technol$\begin{array}{lllll}o g y & 246: & 193-202\end{array}$. DOI:10.1016/j.biortech.2017.07.095.

Grycová, B., I. Koutník, y A. Pryszcz. 2016. "Pyrolysis Process for the Treatment of Food Waste." Bioresource Technology 218: 1203-7. DOI:10.1016/j.biortech.2016.07.064.

Hu, X., y M. Gholizadeh. 2019. “Biomass Pyrolysis: A 
Review of the Process Development and Challenges from Initial Researches up to the Commercialisation Stage." Journal of Energy Che mistry, $\quad 109-43$. DOI:10.1016/j.jechem.2019.01.024.

Kim, Y. H., S. Min Lee, H. Woo Lee, y J. Won Lee. 2012. "Physical and Chemical Characteristics of Products from the Torrefaction of Yellow Poplar (Liriodendron Tulipifera)." Bioresource Technology 116:120-25. DOI:10.1016/j.biortech.2012.04.033.

Klug, M. 2012. “Pirólisis, Un Proceso Para Derretir La Biomasa." Revista de Química PUCP 26: $1-2$.

Kumar, A., y S. R. Samadder. 2017. “A Review on Technological Options of Waste to Energy for Effective Management of Municipal Solid Waste." Waste Management 69: 407-22. DOI:10.1016/j.wasman.2017.08.046.

Kwon, E. E., S. Kim, y J. Lee. 2019. "Pyrolysis of Waste Feedstocks in CO 2 for Effective Energy Recovery and Waste Treatment." Journal of CO2 Utilization 31 (2): 173-80. DOI:10.1016/j.jcou.2019.03.015.

Li, Y., L. W. Zhou, y R .Z. Wang. 2017. "Urban Biomass and Methods of Estimating Municipal Biomass Resources." Renewable and Sustainable Energy Reviews 80 (12): 1017-30. DOI:10.1016/J.RSER.2017.05.214.

Lombardi, L., E. Carnevale, y A. Corti. 2015. "A Review of Technologies and Performances of Thermal Treatment Systems for Energy Recovery from Waste." Waste Management 37 : $26-44$.

DOI:10.1016/j.wasman.2014.11.010.

Martínez-Ángel, J. D., R. A. Villamizar-Gallardo, y O. O. Ortíz-Rodríguez. 2015. "Characterization and Evaluation of Cocoa (Theobroma Cacao L.) Pod Husk as a Renewable Energy Source.” Agrociencia 49(3).

Mattos, C., M. C. C. Veloso, G. A. Romeiro, y E. Folly. 2019. "Biocidal Applications Trends of Bio-
Oils from Pyrolysis: Characterization of Several Conditions and Biomass, a Review." Journal of Analytical and Applied Pyrolysis 139: 1-12. DOI:10.1016/j.jaap.2018.12.029.

Mayer, F., R. Bhandari, y S. Gäth. 2019. “Critical Review on Life Cycle Assessment of Conventional and Innovative Waste-to-Energy Technologies." Science of the Total Environment $672: \quad 708-21$. DOI:10.1016/j.scitotenv.2019.03.449.

Medina, O. L. E., y O. I. N. Medina. 2018. "Prototipo Autotérmico Móvil Para Producción de Biocarbón Con Biomasa de Esquilmos de Aguacate." Revista Terra Latinoamericana 36 (2): 121. DOI:10.28940/terra.v36i2.217.

Oliveira, F. R., A. K. Patel, D. P. Jaisi, S. Adhikari, y H. Lu. 2017. "Environmental Application of Biochar : Current Status and Perspectives." Bioresource Technology 246 (2): 110-122. DOI:10.1016/j.biortech.2017.08.122.

Penedo, M. M., O. Giselle Giralt, Y. Bertrán Guilarte, L. Ana Sánchez del Campo, y H. J. Falcón. 2008. "Pirólisis De Bagazo De Caña A Escala De Laboratorio, Parte Ii: Caracterización De Productos Líquidos De Pirólisis.” Tecnología Química 27 (3): 35-45.

Pérez, J., D. Borge, y J. Agudelo. 2010. “Biomass Gasification Process: Theoretical and Experimental Studies a Review | Proceso de Gasificación de Biomasa: Una Revisión de Estudios Teórico -Experimentales.” Revista Facultad de Ingenieria (52): 95-107.

Rehrah, D., R. R. Bansode, O. Hassan, y M. Ahmedna. 2016. "Physico-Chemical Characterization of Biochars from Solid Municipal Waste for Use in Soil Amendment." Journal of Analytical and Applied Pyrolysis 118: 42-53. DOI:10.1016/j.jaap.2015.12.022.

Romero, M. L. M. , D. M. A. Cruz, y V. F. E. Sierra. 2016. "Effect of Temperature on Energy Potential of Pyrolysis Products from Oil Palm Shells." Tecnura 20 (48): 89-99. 
DOI:10.14483/udistrital.jour.tecnura.2016.2. a06.

Sindhu, R., E. Gnansounou, S. Rebello, P. Binod, S. Varjani, I. Shekhar Thakur, R. B. Nair, y A. Pandey. 2019. "Conversion of Food and Kitchen Waste to Value-Added Products." Journal of Environmental Management 241
( 2 ):
$619-30$.

DOI:10.1016/j.jenvman.2019.02.053.

Sipra, A. T., N. Gao, y H. Sarwar. 2018. "Municipal Solid Waste (MSW) Pyrolysis for Bio-Fuel Production: A Review of Effects of MSW Components and Catalysts." Fuel Processing Technology 175 (10): 131-47. DOI:10.1016/j.fuproc.2018.02.012.

Sizmur, T., T. Fresno, G. Akgül, H. Frost, y E. Morenojiménez. 2017. "Bioresource Technology Biochar Modification to Enhance Sorption of Inorganics from Water." Bioresource Techno logy $246: \quad 34-47$. DOI:10.1016/j.biortech.2017.07.082.

Sorum, L., M. G. Gronli, y J. E. Hustad. 2001. "Pyrolysis Characteristics and Pyrolysis Characteristics and Kinetics of Municipal Solid Wastes."Fuel 80 (9): 1217-27. DOI:10.1016/S0016-2361(00)00218-0.

Stephen, J. L. y B. Periyasamy. 2018. "Innovative Developments in Biofuels Production from Organic Waste Materials: A Review." Fuel $214 \quad\left(\begin{array}{ll}1 & 1\end{array}\right): \quad 623-33$. DOI:10.1016/j.fuel.2017.11.042.

Suárez-Hernández, L., A. Ardila-A, y R. BarreraZapata. 2017. "Morphological and Physicochemical Characterization of Biochar Produced by Gasification of Selected Forestry Species" 26 (46): 123-30.

Sukumar, V., V. Manieniyan, R. Senthilkumar, y S. Sivaprakasam. 2020. "Production of Bio Oil from Sweet Lime Empty Fruit Bunch by Pyrolysis." Renewable Energy 146: 309-15. DOI:10.1016/j.renene.2019.06.156.

Tirapanampai, C., W. Phetwarotai y N. Phusunti.
2019. "Effect of Temperature and the Content of $\mathrm{Na} 2 \mathrm{CO} 3$ as a Catalyst on the Characteristics of Bio-Oil Obtained from the Pyrolysis of Microalgae." Journal of Analytical and Applied Pyrolysis, 104644 . DOI:10.1016/j.jaap.2019.104644.

Tripathi, Manoj, J. N. Sahu, y P. Ganesan. 2016. "Effect of Process Parameters on Production of Biochar from Biomass Waste through Pyrolysis: A Review." Renewable and Sustainable Energy Reviews 55: 467-81. DOI:10.1016/j.rser.2015.10.122.

Wang, Y. y R. Liu. 2017. “Comparison of Characteristics of Twenty-One Types of Biochar and Their Ability to Remove Multi-Heavy Metals and Methylene Blue in Solution." Fuel Processing Technology 160: 55-63. DOI:10.1016/j.fuproc.2017.02.019.

Watts, D. W., P. Randolph, J. M. Novak, R. Ravella, M. R. Reddy, R. R. Bansode, M. Ahmedna, O. A. Hassan, y D. Rehrah. 2017. "Effect of Biochars Produced from Solid Organic Municipal Waste on Soil Quality Parameters." Journal of Environmental Management $192: \quad 271-80$. DOI:10.1016/j.jenvman.2017.01.061.

Zaman, C. Z., K. Pal, W. A. Yehye, S. Sagadevan, S. Tawab Shah, G. Abimbola Adebisi, E. Marliana, R. Faijur Rafique, y R. Bin Johan. 2017. "Pyrolysis: A Sustainable Way to Generate Energy from Waste." In Pyrolysis. DOI:10.5772/intechopen.69036. 